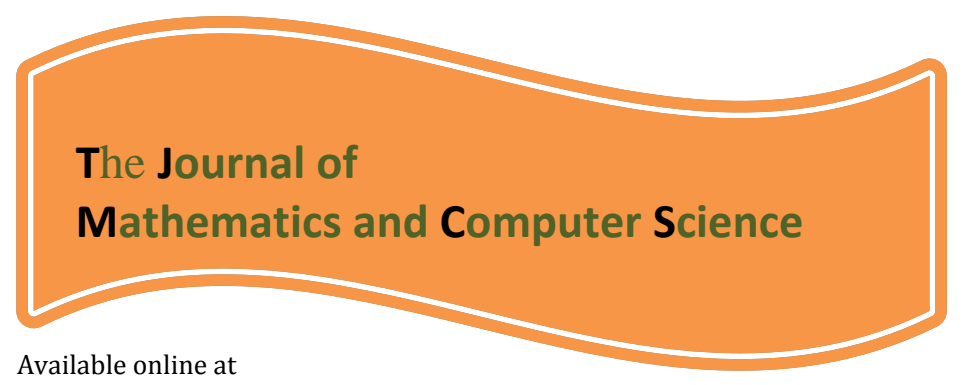

http://www.TIMCS.com

The Journal of Mathematics and Computer Science Vol .2 No.3 (2011) 425-430

\title{
On generalized fractional flux advection-dispersion equation and Caputo derivative
}

\author{
A. Golbabai*1 , K. Sayevand \\ School of Mathematics, Iran University of Science and Technology, \\ P. 0. Box, 16846-13114, Tehran, Iran.
}

Received: September 2010, Revised: November 2010

Online Publication: January 2011

\begin{abstract}
In this study, the homotopy perturbation method is used to solve fractional flux advectiondispersion equation. The problem is formulated in the Caputo sense. The results reveal that the present method is very effective and convenient.
\end{abstract}

Keywords: Caputo derivative, Homotopy perturbation method, fractional flux advectiondispersion equation.

\section{Introduction}

The fractional calculus (calculus of integrals and derivatives of any arbitrary real or complex order) may be considered an old topic (1695). Since that time the fractional calculus has drawn the attention of many researchers. In recent years, fractional calculus has played a significant role in many areas of science and engineering and it has been developed progressively up to now. For an interesting history and more scientific applications of fractional calculus, see [1].

\footnotetext{
$1 *$ Corresponding author. Tel., fax: +982173225417.

E-mail addresses: golbabai@iust.ac.ir, ksayehvand@iust.ac.ir.
} 
Finding approximate or exact solutions of fractional differential equations is an important task. During the last decade, a promising analytic technique is called the homotopy perturbation method (HPM), first proposed by He [3], has successfully been applied to solve many types of linear and nonlinear functional equations.

In this paper, we consider fractional flux advection-dispersion equation in the following form [2]

$$
\frac{\partial \varepsilon(\bar{x}, t)}{\partial t}=-v \frac{\partial \varepsilon(\bar{x}, t)}{\partial t}+D \frac{\partial}{\partial \bar{x}}\left(\frac{1+\eta}{2} \frac{\partial^{\alpha-1} \varepsilon(\bar{x}, t)}{\partial \bar{x}^{\alpha-1}}+\frac{1-\eta}{2} \frac{\partial^{\alpha-1} \varepsilon(\bar{x}, t)}{\partial(-\bar{x})^{\alpha-1}}\right), \quad D, v \in R,-1 \leq \eta \leq 1
$$

Our aim to establish an effective way for solving Eq. (1). To achieve this HPM is used to solve this problem. Results show that proposed theoretical analysis is accurate.

The paper is organized as follows. Section 2 gives notations and basic definitions. In Section 3 we will consider the homotopy perturbation method for solving fractional differential equations. In Section 4 we will consider the one dimensional fractional flux advection-dispersion equation. Finally, Section 5 is devoted to the discussions and conclusions.

\section{Basic definitions}

This section deals with some preliminaries and notations regarding fractional calculus. For more details see[1].

Definition 1. A real function $f(x), x>0$, is said to be in the space $C_{\alpha}, \alpha \in R$ if there exists a real number $p(>\alpha)$, such that $f(x)=x^{p} f_{1}(x)$, where $f_{1}(x) \in C[0, \infty)$.

Definition 2. A function $f(x), x>0$, is said to be in the space $C_{\alpha}^{m}, m \in Z$, if $f^{(m)} \in C_{\alpha}$.

Definition 3. The (left sided) Riemann-Liouville fractional integral of order $\alpha \geq 0$ of a function $f \in C_{\alpha}$ is defined as

$$
\begin{aligned}
I_{t}^{\alpha} f(t) & =\frac{1}{\Gamma(\alpha)} \int_{0}^{t} \frac{f(\tau)}{(t-\tau)^{1-\alpha}} d \tau, \alpha>0, t>0, \\
I_{t}^{0} f(t) & =f(t)
\end{aligned}
$$

where $\Gamma(\alpha)$ is the well-known Gamma function.

Definition4. The (left sided) Caputo fractional derivative of $f, f \in C_{-1}^{m}, m \in Z$ of order $\alpha$ is defined as

$$
D_{* t}^{\alpha} f(t)=\left\{\begin{array}{cr}
I_{t}^{m-\alpha} f^{(m)}(t) & m-1<\alpha<m, m \in Z, \\
\frac{d^{m}}{d t^{m}} f(t) & m=\alpha .
\end{array}\right.
$$




\section{Homotopy perturbation method and Caputo derivative}

To illustrate the basic ideas of the HPM and fractional differential equations, we consider the following equation

$$
D_{*_{t}}^{\alpha} \varepsilon(\bar{x}, t)=v(\bar{x}, t)-L \varepsilon(\bar{x}, t)-N \varepsilon(\bar{x}, t), \quad \bar{x} \in R^{n},
$$

where $L$ is a linear operator while $N$ is a nonlinear operator, $v$ is a known analytical function and $D_{*_{t}}{ }^{\alpha}$ denotes the fractional differential derivative in the Caputo sense, subject to the initial conditions

$$
\varepsilon^{i}(0, \overline{0})=c_{i}, i=0,1, \ldots, n-1,
$$

where $c_{i}$ are arbitrary constants.

In view of He's homotopy perturbation method, we can construct the following simple homotopy

$$
(1-p) D_{*_{t}}^{\alpha} \varepsilon(\bar{x}, t)+p\left(D_{*_{t}}{ }^{\alpha} \varepsilon(\bar{x}, t)-v(\bar{x}, t)+L \varepsilon(\bar{x}, t)+N \varepsilon(\bar{x}, t)\right)=0, \quad p \in[0,1],
$$

or

$$
D_{*_{t}}{ }^{\alpha} \varepsilon(\bar{x}, t)+p(-v(\bar{x}, t)+L \varepsilon(\bar{x}, t)+N \varepsilon(\bar{x}, t))=0, \quad p \in[0,1]
$$

The homotopy parameter $p$ always changes from zero to unity. In case $p=0$, Eq. (7) or Eq. (8) becomes

$$
D_{*_{t}}{ }^{\alpha} \varepsilon(\bar{x}, t)=0
$$

where $p=1$, Eq. (7) or Eq. (8) turns out to be the original fractional differential equation. In view of homotopy perturbation method, we use the homotopy parameter $p$ to expand the solution in the following form

$$
\varepsilon(\bar{x}, t)=\varepsilon_{0}(\bar{x}, t)+p \varepsilon_{1}(\bar{x}, t)+p^{2} \varepsilon_{2}(\bar{x}, t)+\ldots .
$$

For nonlinear problems, let us set $N \varepsilon(\bar{x}, t)=S(\bar{x}, t)$. Substituting Eq. (10) into Eq. (8) or Eq. (7) and equating the terms with identical power of $p$, we can obtain a series of equations of the form

$$
\begin{aligned}
& p^{0}: D_{*_{t}}^{\alpha} \varepsilon_{0}(\bar{x}, t)=0 \\
& p^{1}: D_{*_{t}}^{\alpha} \varepsilon_{1}(\bar{x}, t)=-L \varepsilon_{0}(\bar{x}, t)-S_{0}\left(\varepsilon_{0}(\bar{x}, t)\right)+v(\bar{x}, t), \\
& p^{2}: D_{*_{t}}^{\alpha} \varepsilon_{2}(\bar{x}, t)=-L \varepsilon_{1}(\bar{x}, t)-S_{1}\left(\varepsilon_{0}(\bar{x}, t), \varepsilon_{1}(\bar{x}, t)\right), \\
& p^{3}: D_{*_{t}}^{\alpha} \varepsilon_{3}(\bar{x}, t)=-L \varepsilon_{2}(\bar{x}, t)-S_{2}\left(\varepsilon_{0}(\bar{x}, t), \varepsilon_{1}(\bar{x}, t), \varepsilon_{2}(\bar{x}, t)\right), \\
& \vdots \ldots
\end{aligned}
$$


The functions $S_{0}, S_{1}, S_{2}, \ldots$ satisfy the following equation

$$
S\left(\varepsilon_{0}(\bar{x}, t)+p \varepsilon_{1}(\bar{x}, t)+p^{2} \varepsilon_{2}(\bar{x}, t)+\ldots\right)=\sum_{i=0}^{\infty} S_{i} p^{i}
$$

Where

$$
S_{i}=S_{i}\left(\varepsilon_{0}, \varepsilon_{1}, \ldots, \varepsilon_{i}\right)
$$

Applying the operator $I_{t}^{\alpha}$ on the both sides of the Eqs. (11), with considering the initial conditions, the first few terms of the HPM can be given by

$$
\begin{aligned}
& \varepsilon_{0}(\bar{x}, t)=\sum_{i=0}^{n-1} c_{i} \frac{t^{i}}{i !} \\
& \varepsilon_{1}(\bar{x}, t)=-I_{t}^{\alpha}\left(L \varepsilon_{0}(\bar{x}, t)\right)-I_{t}^{\alpha} S_{0}\left(\varepsilon_{0}(\bar{x}, t)\right)+I_{t}^{\alpha} v(\bar{x}, t), \\
& \varepsilon_{2}(\bar{x}, t)=-I_{t}^{\alpha}\left(L \varepsilon_{1}(\bar{x}, t)\right)-I_{t}^{\alpha} S_{1}\left(\varepsilon_{0}(\bar{x}, t), \varepsilon_{1}(\bar{x}, t)\right), \\
& \varepsilon_{3}(\bar{x}, t)=-I_{t}^{\alpha}\left(L \varepsilon_{2}(\bar{x}, t)\right)-I_{t}^{\alpha} S_{2}\left(\varepsilon_{0}(\bar{x}, t), \varepsilon_{1}(\bar{x}, t), \varepsilon_{2}(\bar{x}, t)\right), \\
& \vdots \ldots
\end{aligned}
$$

Hence, we get an accurate approximation in the following form

$$
\varepsilon(\bar{x}, t)=\sum_{i=0}^{\infty} \varepsilon_{i}(\bar{x}, t)
$$

\section{Applications and results}

Consider the following fractional flux advection-dispersion equation [2]

$$
\left\{\begin{array}{c}
\frac{\partial \varepsilon(\bar{x}, t)}{\partial t}=-v \frac{\partial \varepsilon(\bar{x}, t)}{\partial t}+D \frac{\partial}{\partial \bar{x}}\left(\frac{1+\eta}{2} \frac{\partial^{\alpha-1} \varepsilon(\bar{x}, t)}{\partial \bar{x}^{\alpha-1}}+\frac{1-\eta}{2} \frac{\partial^{\alpha-1} \varepsilon(\bar{x}, t)}{\partial(-\bar{x})^{\alpha-1}}\right), \\
\varepsilon(0, t)=f(t), \quad \varepsilon_{\bar{x}}(0, t)=g(t), \quad \varepsilon(\bar{x}, 0)=h(\bar{x}), \quad-1 \leq \eta \leq 1, \quad[\alpha]=m \in Z,
\end{array}\right.
$$

We construct the following homotopy

$$
\frac{\partial \varepsilon(\bar{x}, t)}{\partial t}+p\left(v \frac{\partial \varepsilon(\bar{x}, t)}{\partial t}-D \frac{\partial}{\partial \bar{x}}\left(\frac{1+\eta}{2} \frac{\partial^{\alpha-1} \varepsilon(\bar{x}, t)}{\partial \bar{x}^{\alpha-1}}+\frac{1-\eta}{2} \frac{\partial^{\alpha-1} \varepsilon(\bar{x}, t)}{\partial(-\bar{x})^{\alpha-1}}\right)\right)=0, \quad p \in[0,1]
$$

Assume now that the solution of Eq. (17) to be in the form 


$$
\varepsilon(\bar{x}, t)=\sum_{i=0}^{\infty} \varepsilon_{i}(\bar{x}, t) p^{i}
$$

Substituting (18) into (17) and equating the coefficients of like powers of $p$,we get following set of differential equations

$$
\frac{\partial \varepsilon_{j}}{\partial t}=-v \frac{\partial \varepsilon_{j}}{\partial \bar{x}}+D \frac{\partial}{\partial \bar{x}}\left(\frac{1+\eta}{2} \frac{\partial^{\alpha-1} \varepsilon_{j-1}}{\partial \bar{x}^{\alpha-1}}+\frac{1-\eta}{2} \frac{\partial^{\alpha-1} \varepsilon_{j-1}}{\partial(-\bar{x})^{\alpha-1}}\right), \quad j=1,2, \ldots
$$

Consequently, the solution is

$$
\varepsilon(\bar{x}, t)=\varepsilon(\bar{x}, 0)+\sum_{i=1}^{\infty} \varepsilon_{i}(\bar{x}, t)
$$

Fig.1. Shows solution by using HPM for $t=2, v=D=1, \eta=0, \varepsilon_{0}=e^{x}, \alpha=0.5$.

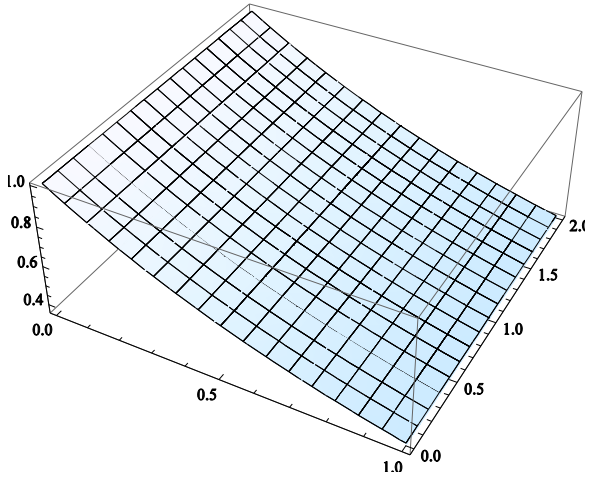

Fig. 1 .

\section{Discussions and conclusion}

In this paper, HPM is applied to solve the fractional flux advection-dispersion equation based on the Caputo definition. Results show that the proposed HPM is powerful and reliable. We have to pointed out that Mathematica has been used for programming and computations in this paper.

\section{References}

[1]. Podlubny, I., “Fractional Differential Equations”, Academic Press, San Diego, 1999.

[2]. Quanzhong, H., Guanhua, H., Hongbin, Z. "A finite element solution for fractional advectiondispersion equation", Advances in water Resources, (2008) 1578-1589. 
A. Golbabai, K. Sayevand/ TJMCS Vol .2 No.3 (2011) 425-430

[3]. He, J. H., "Homotopy perturbation technique”, Computer Methods in Applied Mechanics and Engineering, 178 (1999) 257-262. 